\title{
Loss of genetic accuracy in mutants of the thermoacidophile Sulfolobus acidocaldarius
}

\author{
GREG D. BELL ${ }^{1}$ and DENNIS W. GROGAN ${ }^{1,2}$ \\ ${ }^{1}$ Department of Biological Sciences, University of Cincinnati, Cincinnati, OH 45221-0006, USA \\ ${ }^{2}$ Author to whom correspondence should be addressed (grogandw@email.uc.edu)
}

Received July 24, 2001; accepted October 2, 2001; published online October 30, 2001

\begin{abstract}
Summary To investigate how hyperthermophilic archaea can propagate their genomes accurately, we isolated Sulfolobus acidocaldarius mutants exhibiting abnormally high rates of spontaneous mutation. Our isolation strategy involved enrichment for mutator lineages via alternating selections, followed by screening for the production of spontaneous, 5-fluoroorotate-resistant mutants in micro-colonies. Several candidates were evaluated and found to have high frequencies of $p y r E$ and $p y r F$ mutation and reversion. Neither an increased efficiency of plating of mutants on selective medium, nor the creation of a genetically unstable pyrE allele, could be implicated as the cause of these high frequencies. The strains had elevated frequencies of other mutations, and exhibited certain phenotypic differences among themselves. A large increase in sensitivity to DNA-damaging agents was not observed, however. These properties generally resemble those of bacterial mutator mutants and suggest loss of functions specific to genetic accuracy.
\end{abstract}

Keywords: 5-fluoro-orotic acid, hyperthermophilic archaea, mismatch repair, mutator mutants, spontaneous mutation.

\section{Introduction}

Two generalizations can be made for all unicellular organisms in which genetic accuracy has been examined: (i) rates of error incorporation, measured as spontaneous mutation, are extremely low (Drake et al. 1998), and (ii) achieving these low rates requires several distinct and specialized proteins that cooperate to prevent, recognize and correct structural errors in DNA (Miller 1998). These observations imply that, aside from special situations where high mutation rates may be advantageous (LeClerc et al. 1996, Mao et al. 1997, Sniegowski et al. 1997), genetic fidelity is important for the evolutionary success of microorganisms. On the other hand, mechanisms that cooperate to maintain this fidelity are not required for viability under laboratory conditions. As a result, it has been possible to isolate and study strains in which various accuracy-enforcing functions have been lost by mutation. These strains share the characteristic phenotype that the rate of spontaneous mutation is elevated, and are termed "mutator" mutants.
Most of the known biochemical functions needed for genetic accuracy were first identified by genetic and molecular analysis of Escherichia coli mutator mutants. The corresponding genes have been found to encode subunits of the replicative DNA polymerase complex (dnaE, dnaQ), DNA $\mathrm{N}$-glycosylases (mutM, mutY, nei, nth, ung, vsr), enzymes that destroy oxidized DNA precursors or intracellular superoxide $(m u t T, \operatorname{sod} A, \operatorname{sodB})$, and components of a generalized mismatch repair (MMR) system (mutH, mutL, mutS, uvrD) (Miller 1998). The MMR system of E. coli degrades and resynthesizes one strand of a duplex DNA molecule containing a mispaired base, and in so doing, provides one of the organism's most important defenses against spontaneous mutation. Inactivation of the $m u t S$ or $m u t L$ genes increases spontaneous mutation by a factor of 50-1000, and is the most common cause of high spontaneous mutation in enteric bacteria (LeClerc et al. 1996, Harris et al. 1997). Other organisms utilize related systems, as indicated by the presence of mutS and $m u t L$ homologs in bacterial and eukaryal genomes and similar genetic defects caused by inactivation of these homologs (Eisen 1998, Aravind et al. 1999, Nakagawa et al. 1999). In particular, eukarya have multiple, functionally differentiated homologs of the E. coli proteins, which interact in different combinations to carry out a spectrum of distinct repair functions (Nakagawa et al. 1999).

This otherwise widely conserved family of proteins is apparently rare among archaea, however. To our knowledge, putative $m u t S$ and $m u t L$ genes coincide in only one archaeal genome, that of a Halobacterium species, whereas another mut $S$ homolog occurs without mutL in the genome of a moderately thermophilic methanogen (Smith et al. 1997, $\mathrm{Ng}$ et al. 2000). Sequence analysis has failed to identify either mutS or mutL homologs in the complete genomes of several hyperthermophilic archaea, including members of both the euryarchaeota and the crenarchaeota (reviewed in Grogan 2000). This absence seems particularly unusual in view of the accelerated formation of pre-mutagenic lesions expected at the extremely high growth temperatures of these archaea (Lindahl 1993), and the fact that bacteria with similar growth temperatures have both mutS and mutL homologs (Deckert et al. 1998, Nelson et al. 1999). Furthermore, experiments confirm that 
hyperthermophilic archaea can replicate their genomes accurately. Loss-of-function mutations occur in two Sulfolobus acidocaldarius uridine $5^{\prime}$-monophosphate (UMP)-biosynthetic genes at rates close to those of biosynthetic genes in MMRproficient E. coli strains (Jacobs and Grogan 1997). Although the corresponding genes are inactivated at higher rates in S. solfataricus, the difference can be explained by the frequent transposition of insertion sequence (IS) elements in that species (Martusewitsch et al. 2000).

Available data thus indicate that hyperthermophilic archaea lack homologs of the MutS and MutL proteins found in all bacteria and eukarya, yet those species that have been assayed genetically do not mimic the high mutation rates of bacterial or eukaryotic mutants lacking MMR or other fidelity-enforcing mechanisms. This raises the question as to what proteins actually enforce genetic accuracy in hyperthermophilic archaea. Although the availability of genomic sequences facilitates efforts to identify such proteins, it must be recognized that many relevant genes in these archaea may continue to escape identification because of insufficient sequence similarity to known genes. This divergence, combined with the narrowly genetic function of accuracy-enforcing genes, places strategic importance on applying suitable experimental genetic methods as a route to investigating the basis of genetic accuracy in hyperthermophilic archaea. In the present study, we demonstrate the feasibility of direct functional approaches to this question by isolating and characterizing mutator mutants of $S$. acidocaldarius.

\section{Materials and methods}

\section{Strains and growth conditions}

Sulfolobus acidocaldarius strains DG185 (ATCC33909) and DG64 are wild-type and pyrB4, respectively. Strain DG64 was isolated by replica plating and is deficient in aspartate carbamyl transferase (Grogan and Gunsalus 1993). All growth media contained a mineral mixture (Grogan and Gunsalus 1993) supplemented with $0.2 \%$ carbon source (D-xylose or Dextrin-10 (Fluka, Buchs, Switzerland)), 0.1\% nitrogen source (L-glutamine plus acid-hydrolyzed casein ("casamino acids"), or enzymatically digested casein ("tryptone")). Media were solidified, as necessary, with $0.65 \%$ gellan gum (Grogan and Gunsalus 1993). Supplements were added to yield the following concentrations (in $\mathrm{mg} \mathrm{l}^{-1}$ ): yeast extract, 500; DL-ethionine, 250; 5-fluoro-orotic acid (FOA), 50; orotic acid, 30; and uracil, 20. Cultivation in microdilution plates and longterm storage were as previously described (Grogan 1995). All incubation temperatures were $75^{\circ} \mathrm{C}$ unless otherwise noted.

\section{Mutator isolation}

Four suspensions of DG64 cells $\left(3.5 \times 10^{9}\right.$ cells each) were treated with the alkylating agent $N$-methyl- $N^{\prime}$-nitro- $N$-nitrosoguanidine (MNNG) (two each at 0.2 and $0.4 \mathrm{mg} \mathrm{ml}^{-1}$ ) for $30 \mathrm{~min}$ at $37{ }^{\circ} \mathrm{C}$ as previously described (Bernander et al. 2000). In all subsequent steps, the four cell populations, designated Populations I-IV, were processed in parallel. The cells were washed free of mutagen and incubated in permissive xylose-tryptone-uracil (XT.ura) medium for $15 \mathrm{~h}$. About $4 \times$ $10^{6}$ cells were then transferred to fresh XT.ura medium and propagated for 15 generations at $70{ }^{\circ} \mathrm{C}$ (total of three $1: 30$ inoculations). Aliquots of the resulting four cultures were then plated on XT.ura medium plus FOA (XT.ura.foa) and incubated for 6 to 8 days, yielding an average of 350 colonies per plate. About 2000 colonies were pooled by resuspension in sterile buffer using a glass spreader, and an aliquot (containing about $10^{8}$ cells) was transferred to XT.ura medium and propagated for 15 generations at $80{ }^{\circ} \mathrm{C}$. Aliquots of these cultures were plated on dextrin-glutamine-casamino acid-orotate (DG.caa.oro) medium at $80{ }^{\circ} \mathrm{C}$ to select $\mathrm{Oro}^{+}$clones. About 2000 of the resulting colonies were pooled.

The above four-step sequence (outgrowth, FOA-resistance $\left(\mathrm{Foa}^{\mathrm{R}}\right.$ ) selection, outgrowth, $\mathrm{Oro}^{+}$selection) was performed three times for each of the four mutagenized populations. Aliquots of the resulting pools of $\mathrm{Oro}^{+}$colonies were then grown for an additional 10 generations in liquid DG.caa.oro medium to deplete these populations of residual $\mathrm{Foa}^{\mathrm{R}}$ cells. Aliquots of the resulting cultures were plated on XT.ura medium to yield 300-400 colonies per plate. After incubating the plates for 3 days, $1 \mathrm{mg}$ FOA was added to each plate as small droplets of a concentrated solution deposited around the extreme margin of the plate. After these droplets had soaked into the medium, the plates were incubated for an additional 7 days. Colonies that had enlarged to normal size (as judged by comparison with control plates not receiving FOA) were marked with a spot on the back of the plate. The plates were incubated for an additional 3 days and reexamined. Colonies that had enlarged during these last 3 days were streaked for isolation on selective (XT.ura.foa) medium.

\section{Genetic assays}

The resulting clones were evaluated for forward and reverse mutation of the pyrE and $p y r F$ genes by picking several isolated colonies of each candidate and growing them in $200 \mu \mathrm{l}$ of $\mathrm{XT}$.ura medium to a density of about $5 \times 10^{8}$ cells $\mathrm{ml}^{-1}$. Reversion was assayed by spreading $50 \mu \mathrm{l}$ of each culture onto solid xylose-glutamine-casamino acid (XG.caa) medium supplemented with orotic acid. Clones producing large numbers of $\mathrm{Oro}^{+}$revertants in all replicates were tested for forward mutation in a similar manner, except that the test was performed on one of the $\mathrm{Oro}^{+}$revertants and the cells were plated on XT.ura.foa medium. The $\mathrm{Foa}^{\mathrm{R}}$ and $\mathrm{Oro}^{+}$derivatives of 27 candidates assayed for forward and reverse mutation were stored at $-70{ }^{\circ} \mathrm{C}$.

Phenotypic reversion of the pyrB4 allele was assayed using several independent cultures of the $\mathrm{Oro}^{+}$derivative of each strain. The cells were washed free of medium and plated on XG.caa medium, which lacks a pyrimidine source. Mutation to ethionine resistance was assayed by plating independent cultures on XT.ura medium supplemented with DL-ethionine. In both cases ( $p y r B$ reversion and ethionine resistance), plates were incubated for at least 10 days to ensure formation of visible colonies.

Nonselected mutation was assayed by inoculating liquid 
xylose-glutamine-uracil-yeast extract medium (XG.ura.ye) with an isolated colony of the candidate or control strain. The resulting cultures were propagated at $70{ }^{\circ} \mathrm{C}$ for 30 generations by repeated subculturing of inocula containing about $3 \times 10^{8}$ cells. Aliquots of the resulting liquid cultures were plated on XG.ura.ye medium at $70{ }^{\circ} \mathrm{C}$; the resulting colony patterns were replica plated (Grogan 1995) to XG.ura.ye at $70{ }^{\circ} \mathrm{C}$ (reference plates), XG.ura plates incubated at $70{ }^{\circ} \mathrm{C}$ (to detect auxotrophs) and XG.ura.ye plates incubated at $83^{\circ} \mathrm{C}$ (to detect thermosensitive (Ts) mutants). When the replicas were compared, care was taken to retain all colonies showing any sign of differential growth for subsequent testing. This confirmatory testing consisted of making one short $(2-3 \mathrm{~cm})$ streak of the colony on two test plates, which were incubated under the corresponding scoring conditions. Clones that grew on both plates were disqualified. In the case of one culture, these disqualified clones represented $50 \%$ of the colonies picked from the reference plates, whereas in all other cases the proportion disqualified was $75 \%$ or greater. This suggests that few auxotrophic or Ts colonies went undetected in the replica plating analysis.

Efficiencies of plating (EOP) of S. acidocaldarius strains were assayed using clonally pure cultures of the $\mathrm{Foa}^{\mathrm{R}}$ derivatives grown in nonselective (XT.ura) medium to a density of about $3 \times 10^{8}$ cells $\mathrm{ml}^{-1}$. Two aliquots of dilutions of these cultures were each plated on both XT.ura and XT.ura.foa media. The mean number of colonies forming on the nonselective (XT.ura) plates was divided by the mean on the selective (XT.ura.foa) plates to yield the relative EOP.

\section{Determination of sensitivities}

Chemical mutagens were evaluated by serial 1:2 dilutions of the compound in liquid XT.ura medium, which were inoculated with about $3 \times 10^{6}$ cells. The resulting test cultures (200 $\mu \mathrm{l}$ each) were incubated for 3 days and growth was scored visually to determine the minimum inhibitory concentration (MIC). Each MIC determination was performed four times and the means were determined. Sensitivity to UV radiation was tested by plating three equal aliquots of a dilution onto three identical nonselective (XT.ura) plates. One plate was incubated without irradiation, whereas the other two were irradiated with a germicidal lamp. Irradiated plates were shielded from light until after incubation to prevent photoreactivation (Wood et al. 1997). Colony counts were used to calculate the fraction of cells surviving each UV dose.

\section{Results}

\section{Mutator isolation}

To increase the chances of recovering mutators, we designed conditions for enriching mutator lineages in a population of S. acidocaldarius, as well as a method of screening large numbers of $S$. acidocaldarius clones for mutant production. Our isolation scheme consisted of three stages: (i) mutagenesis and phenotypic expression; (ii) mutator enrichment; and (iii) colony scoring. Because Stage ii involved many generations of growth in liquid medium, four mutagenized populations of the parent strain were processed in parallel to allow different mutator mutants to be identified even if they did not exhibit clearcut phenotypic differences.

Enrichment was based on the principle that mutator lineages have a long-term selective advantage in unstable (i.e., repeatedly changing) environments, reflecting the production of subsequently successful mutants at a higher rate than non-mutator lineages (Mao et al. 1997). We produced such an environment by applying a series of alternating selections with intervening periods of nonselective growth. The two primary selections used were (i) 5-fluoro-orotic acid (FOA) plus uracil, which selects loss of pyrE or pyrF function (Grogan and Gunsalus 1993), and (ii) orotic acid as sole pyrimidine source, which selects restoration of $p y r E$ or $p y r F$ gene function. In wild-type cells, the loss of $p y r E$ or $p y r F$ blocks the de novo pathway of UMP biosynthesis, making the cells absolutely dependent on exogenous pyrimidines (e.g., uracil) for growth. As a result, phenotypic reversion of the mutation can be selected by omitting pyrimidines from the medium. This selection for reversion was not feasible in the present study, however, because de novo pyrimidine synthesis was already inactivated in the parental strain by the pyrB4 allele (Grogan and Gunsalus 1993). Instead, selection for growth on orotic acid as the sole pyrimidine supplement (the $\mathrm{Oro}^{+}$phenotype) was used to select specifically for restoration of $p y r E$ or $p y r F$ function; this occurs independently of the status of genes controlling earlier biosynthetic steps, such as pyrB (Grogan and Gunsalus 1993). To provide additional changes in growth conditions, incubation temperature was also manipulated in the enrichment, although the selective impact of this parameter was not assessed. It should be noted that, because the $\mathrm{Oro}^{+}$selection reversed the results of the prior FOA selection, the enrichment cycle could be repeated indefinitely.

After subjecting the four parallel populations to three complete cycles of forward and reverse selection, we scored the ability of the resulting $\mathrm{Oro}^{+}$clones to produce $\mathrm{Foa}^{\mathrm{R}}$ mutants. Our screening method was analogous to the papillation assay used to identify $E$. coli clones that form lactose fermentation ability $\left(\mathrm{Lac}^{+}\right)$revertants at abnormal rates (Nghiem et al. 1988). However, whereas the $\mathrm{Lac}^{+}$papillation assay applies nonlethal selection and chromogenic scoring to large colonies, our technique applied lethal selection to micro-colonies. The Oro $^{+}$cells (300-400) were spread on the surface of a nonselective plate and incubated until they had formed micro-colonies of about $0.1 \mathrm{~mm}$ diameter. 5-Fluoro-orotic acid was then added and the plates were re-incubated. This FOA addition halted the growth of most micro-colonies at a final size of about $3 \times 10^{5}$ cells. A minority of these micro-colonies continued to enlarge, however, as illustrated in Figure 1.

From the four parallel populations, we scored about 18,000 Oro $^{+}$clones by this method. Of these colonies, 365 (about 2\%) that enlarged relatively late following FOA addition were streaked for isolation on selective (FOA-containing) medium. The resulting clones were evaluated for frequency of reversion to the $\mathrm{Oro}^{+}$phenotype, and a revertant from each was similarly streaked for isolation and evaluated for the frequency of for- 


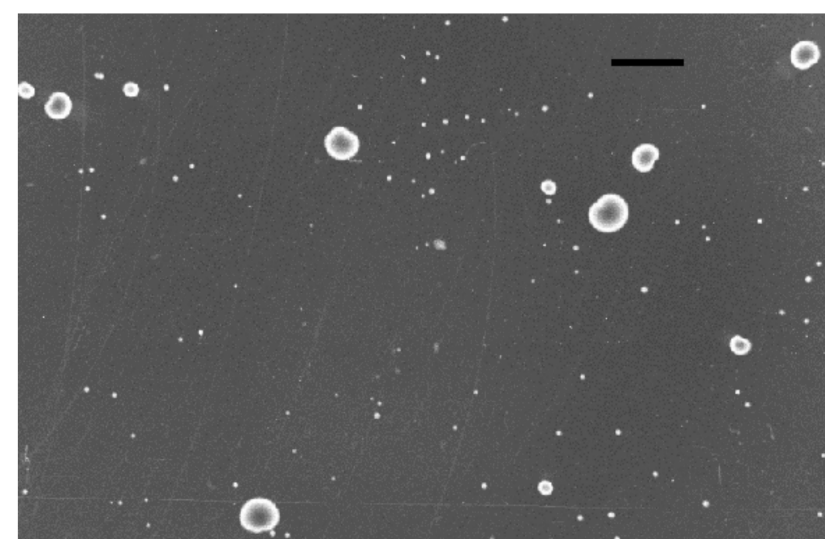

Figure 1. Screening for the formation of mutants in micro-colonies. The photograph shows a xylose-tryptone-uracil (XT.ura) plate spread with cells from the last cycle of enrichment for mutator mutants (see Materials and methods). The FOA was added after a 3-day incubation, followed by an additional 10-day incubation to reveal the growth of FOA-resistant clones. Bar $=5 \mathrm{~mm}$.

ward mutation ( $\mathrm{Foa}^{\mathrm{R}}$ phenotype). Based on three independent determinations of reverse and forward mutation, 27 candidates were retained for further characterization.

\section{Frequencies of selectable mutation}

Frequencies of $\mathrm{Foa}^{\mathrm{R}}$ and $\mathrm{Oro}^{+}$mutants in independent cultures of the 27 candidates are compared with those of the parental strain DG64 in Table 1. Most of these candidates yielded 50-250 times more $\mathrm{Foa}^{\mathrm{R}}$ mutants and 5-50 times more Oro ${ }^{+}$ revertants than did the parental strain. Conceivably, these elevated yields of $\mathrm{Foa}^{\mathrm{R}}$ colonies could reflect a situation in which $\mathrm{Foa}^{\mathrm{R}}$ mutants are detected with low efficiency if they form in the control strains but with high efficiency if they form in altered genetic backgrounds represented by the isolated candidates. To address this possibility, we tested $\mathrm{Foa}^{\mathrm{R}}$ mutants of eight candidates to determine if they plated with much higher efficiency on FOA medium than the $\mathrm{Foa}^{\mathrm{R}}$ mutants of normal S. acidocaldarius strains. The results (Table 2) show that $\mathrm{Foa}^{\mathrm{R}}$ mutants of the control strains had high EOP on FOA medium, and that derivatives of the mutator candidates usually had similar or lower values. Thus, the much higher yields of spontaneous $\mathrm{Foa}^{\mathrm{R}}$ colonies produced by the candidates could not be explained by more efficient detection of mutant cells in the cultures.

The DNA sequence analysis of over 100 spontaneous Foa $^{\mathrm{R}}$ mutants of $S$. acidocaldarius isolated under similar conditions has shown that all of these mutations occur within the $p y r E$ or pyr $F$ genes and that the pyrE mutations dominate, comprising about $95 \%$ of the total (Grogan et al. 2001). As a means of characterizing the effects of repeated forward and reverse selection at these loci, we therefore amplified and sequenced the pyrE gene of the $\mathrm{Oro}^{+}$derivatives of Candidates 2, 7, 8, 10, 11, 12, 22 and 27. This analysis would detect neutral mutations in pyrE that may have become fixed during the isolation and scoring procedures, including the formation of a functional pyrE sequence with an intrinsically higher rate of mutation,
Table 1. Mutation of the pyrE and pyrF genes.

\begin{tabular}{|c|c|c|c|c|}
\hline \multirow{2}{*}{$\begin{array}{l}\text { Candidate } \\
\text { number }\end{array}$} & \multicolumn{2}{|l|}{ Forward } & \multicolumn{2}{|l|}{ Reverse } \\
\hline & $\begin{array}{l}\text { Mutant } \\
\text { frequency }^{1}\end{array}$ & $\begin{array}{l}\text { Relative } \\
\text { factor }^{2}\end{array}$ & $\begin{array}{l}\text { Mutant } \\
\text { frequency }^{1}\end{array}$ & $\begin{array}{l}\text { Relative } \\
\text { factor }^{2}\end{array}$ \\
\hline (DG64) & 1.21 & (1) & 3.30 & (1) \\
\hline 1 & 175 & 144 & 7.1 & 2.2 \\
\hline 2 & 298 & 246 & 23 & 6.9 \\
\hline 3 & 1.0 & 0.8 & 19 & 5.8 \\
\hline 4 & 333 & 275 & 23 & 7.0 \\
\hline 5 & 60 & 50 & 9.1 & 2.8 \\
\hline 6 & 318 & 262 & 81 & 25 \\
\hline 7 & 185 & 152 & 22 & 6.7 \\
\hline 8 & 211 & 174 & 48 & 15 \\
\hline 9 & 381 & 314 & 76 & 23 \\
\hline 10 & 71 & 58 & 1.5 & 0.5 \\
\hline 11 & 141 & 116 & 11 & 3.2 \\
\hline 12 & 598 & 493 & 131 & 40 \\
\hline 13 & 119 & 98 & 286 & 87 \\
\hline 14 & 101 & 83 & 238 & 72 \\
\hline 15 & 101 & 83 & 286 & 87 \\
\hline 16 & 128 & 105 & 57 & 17 \\
\hline 17 & 61 & 50 & 63 & 19 \\
\hline 18 & 175 & 144 & 270 & 82 \\
\hline 19 & 124 & 102 & 151 & 46 \\
\hline 20 & 286 & 235 & 75 & 23 \\
\hline 21 & 111 & 92 & 21 & 6.4 \\
\hline 22 & 233 & 192 & 37 & 11 \\
\hline 23 & 73 & 60 & 16 & 5.0 \\
\hline 24 & 159 & 131 & 14 & 4.1 \\
\hline 25 & 67 & 55 & 159 & 48 \\
\hline 26 & 95 & 78 & 48 & 14 \\
\hline 27 & 187 & 154 & 409 & 124 \\
\hline
\end{tabular}

${ }^{1}$ Mutant colonies per $10^{5}$ cells plated (means of three independent cultures).

2 Relative to strain DG64 under the same conditions.

such as might result from the creation of a new mutational "hot spot." No deviations from the wild-type sequence were found in the resulting eight sequences, indicating that creation of a functional, but genetically unstable, pyrE allele is not a general

Table 2. Relative efficiencies of plating (EOP).

\begin{tabular}{lccc}
\hline Candidate number & Nonselective $^{1}$ & Selective $^{1}$ & Ratio \\
\hline (DG64) & 10.41 & 7.11 & 1.46 \\
(DG185) & 13.2 & 11.93 & 1.11 \\
2 & 0.19 & 0.11 & 1.73 \\
7 & 11.01 & 11.1 & 0.99 \\
8 & 13.91 & 17.21 & 0.81 \\
10 & 11.88 & 19.7 & 0.60 \\
11 & 9.65 & 8.76 & 1.10 \\
12 & 19.91 & 16.28 & 1.22 \\
22 & 1.13 & 0.99 & 1.14 \\
27 & 4.03 & 4.33 & 0.93 \\
\hline
\end{tabular}

1 Viable titers $\left(10^{7} \mathrm{CFU} \mathrm{ml}{ }^{-1}\right)$ of liquid cultures plated on XT.ura medium alone (nonselective) or supplemented with FOA (selective). 
cause of the high frequencies of forward mutation in these strains.

To confirm that increased rates of mutation were not unique to the $p y r E$ and $p y r F$ genes, we assayed spontaneous generation of two other phenotypes in the candidates: prototrophy (i.e., phenotypic reversion of the pyrB4 allele) and resistance to ethionine. As observed previously (Grogan 1995), both of these mutations were relatively rare in the control strains (Table 3). In most of the 27 clones evaluated, however, $p y r B$ reversion, which could include intragenic or extragenic compensatory (i.e., suppressor) mutation, was elevated 10- to 60-fold, whereas mutation to ethionine resistance was elevated 4- to 20-fold (Table 3).

\section{Nonselected mutation}

From the 27 mutator candidates, 10 that yielded high mutant frequencies in most of the selections (Tables 1 and 3), but showed potential differences from each other, were studied in more detail. Eight of these 10 strains were tested for the spontaneous formation of auxotrophic and Ts mutants. An isolated colony of each candidate was grown for about 30 generations at $70{ }^{\circ} \mathrm{C}$ in minimal medium supplemented with $0.05 \%$ yeast extract. The resulting populations of putative mutators (and the two control strains DG64 and DG185) were then tested for the presence of Ts or auxotrophic mutants by replica plating on the same medium at $83{ }^{\circ} \mathrm{C}$ and on medium lacking yeast extract at $70{ }^{\circ} \mathrm{C}$, respectively. The results (Table 4) show that populations of Candidates 8 and 11 contained high frequencies of both Ts and auxotrophic mutants. The frequencies of auxotrophs were about 300 times the mean frequency of the control populations, whereas Ts mutants were about 15 -fold more abundant. The fact that both Ts and auxotrophic phenotypes were recovered and that neither are selected under these conditions provided additional evidence for general elevation of spontaneous mutation rates in Candidates 8 and 11. The fact that two independent cultures yielded similar frequencies further indicates that these results are not explained as rare "jackpot" events. Candidate 22 showed a frequency of auxotrophs about 11 times the mean value for the control strains (Table 4).

\section{Effects of DNA-damaging agents}

To determine whether the observed loss of genetic fidelity was accompanied by a general loss of DNA repair capacity, the sensitivities of 10 candidates to DNA-damaging agents were

Table 3. Frequencies of other mutations.

\begin{tabular}{|c|c|c|c|c|c|c|}
\hline \multirow[t]{2}{*}{ Candidate number } & \multicolumn{3}{|l|}{ pyrB reversion } & \multicolumn{3}{|l|}{ Ethionine resistance } \\
\hline & Mutant frequency $^{1}$ & Standard deviation ${ }^{1}$ & Relative factor ${ }^{2}$ & Mutant frequency $^{1}$ & Standard deviation ${ }^{1}$ & Relative factor ${ }^{2}$ \\
\hline Control $^{3}$ & 1.8 & 0.1 & (1) & 3 & 2.7 & (1) \\
\hline 1 & 13 & 11.4 & 7 & 1.8 & 3.1 & 0.6 \\
\hline 2 & 105 & 28.2 & 58 & 688 & 578 & 228 \\
\hline 3 & 34.2 & 10.1 & 19 & 6 & 0.1 & 2 \\
\hline 4 & 89.7 & 68.7 & 50 & 9.8 & 7.6 & 3.2 \\
\hline 5 & 6.2 & 10.8 & 3 & 5.1 & 8.8 & 1.7 \\
\hline 6 & 30.2 & 6 & 17 & 3.7 & 6.4 & 1.2 \\
\hline 7 & 132 & 91.5 & 73 & 45.9 & 17.8 & 15.2 \\
\hline 8 & 66 & 28.5 & 37 & 71.7 & 43.6 & 23.8 \\
\hline 9 & 49.2 & 49.1 & 27 & 21.4 & 16.8 & 7.1 \\
\hline 10 & 18.7 & 12.6 & 10 & 12.6 & 7.8 & 4.2 \\
\hline 11 & 53.6 & 39.3 & 30 & 13.2 & 5.6 & 4.4 \\
\hline 12 & 181 & 49.6 & 101 & 54.3 & 11 & 18 \\
\hline 13 & 81 & 60.2 & 45 & 26.3 & 16.6 & 8.7 \\
\hline 14 & 52.9 & 80 & 29 & 16.6 & 28.7 & 5.5 \\
\hline 15 & 28.4 & 15.3 & 16 & 13.1 & 22.6 & 4.3 \\
\hline 16 & $<0.6$ & 10.9 & $<0.3$ & 13.4 & 16.4 & 4.4 \\
\hline 17 & 43.6 & 11.8 & 24 & 13.2 & 6.4 & 4.4 \\
\hline 18 & 11.9 & 11.4 & 7 & 8.6 & 4.2 & 2.9 \\
\hline 19 & 110 & 40.9 & 61 & 14.5 & 3.6 & 4.8 \\
\hline 20 & 15.4 & 17.1 & 9 & 19.3 & 33.4 & 6.4 \\
\hline 21 & 42.7 & 27.4 & 24 & 19.1 & 33.2 & 6.3 \\
\hline 22 & 57.6 & 30.5 & 32 & 85 & 60 & 28.2 \\
\hline 23 & 158 & 205 & 88 & 32.8 & 31.7 & 10.9 \\
\hline 24 & 50.4 & 28.7 & 28 & 13.1 & 22.7 & 4.3 \\
\hline 25 & 2 & 3.4 & 1 & 4.3 & 7.4 & 1.4 \\
\hline 26 & 31.8 & 43.7 & 18 & 4 & 7 & 1.3 \\
\hline 27 & 141 & 62.6 & 78 & 2059 & 3567 & 683 \\
\hline
\end{tabular}

${ }^{1}$ Means and standard deviations per $10^{8}$ cells plated $(n=3)$.

${ }^{2}$ Relative to the control strain under the same conditions.

${ }^{3}$ For $p y r B$ reversion, the control strain was DG64; for ethionine resistance, it was DG185. 
Table 4. Frequencies of nonselected mutants determined by replica plating.

\begin{tabular}{llcl}
\hline $\begin{array}{l}\text { Candidate } \\
\text { number }\end{array}$ & $\begin{array}{l}\text { Colonies } \\
\text { scored }\end{array}$ & $\begin{array}{l}\text { Auxotrophs } \\
\text { confirmed }(\%)\end{array}$ & $\begin{array}{l}\text { Ts mutants } \\
\text { confirmed }(\%)\end{array}$ \\
\hline (DG64) & 1620 & 0 & $1(0.06)$ \\
(DG185) & 1530 & $1(0.07)$ & 0 \\
2 & 1590 & 0 & 0 \\
7 & 1280 & 0 & 0 \\
8 & 1050 & $103(9.8)$ & $30(0.29)$ \\
10 & 1750 & 0 & $2(0.11)$ \\
11 & 1420 & $123(8.7)$ & $70(0.49)$ \\
12 & 1720 & 0 & 0 \\
22 & 1470 & $6(0.4)$ & 0 \\
27 & 1400 & 0 & $1(0.07)$ \\
\hline
\end{tabular}

compared to the corresponding sensitivities of the two control strains. The agents included alkylating agents (MNNG and $N$-methyl- $N$-nitrosourea (MNU)), cross-linking agents (cisplatin and butadiene 1,3-diepoxide) and short wavelength UV-light (UV-C). As shown in Table 5, observed sensitivities of the mutator candidates were generally similar to those of the control strains, with the possible exception of Candidate 10.

\section{Discussion}

Nucleotide excision repair (NER) and mismatch repair (MMR) are two of the most versatile and evolutionarily conserved strategies of DNA repair. To our knowledge, the only organisms in which complete sets of NER and MMR genes cannot be identified are archaea with optimal growth temperatures of about $80^{\circ} \mathrm{C}$ or higher (Grogan 2000). This situation, which occurs in euryarchaeota as well as crenarchaeota, cannot be explained by an intrinsic inability of either repair system to function at extremely high temperatures, as complete sets of both NER and MMR genes occur in the genomes of Thermotoga maritima and Aquifex aeolicus, two bacteria that grow optimally at about 80 and $95^{\circ} \mathrm{C}$, respectively (Deckert et al. 1998, Nelson et al. 1999).

The absence of both MutS and MutL homologs from hyperthermophilic archaea raises fundamental questions regarding the universality of accurate DNA replication and repair, and the evolution of fidelity mechanisms among the three domains of life. Quantitative assays of spontaneous loss-of-function mutations confirm highly accurate replication and repair in $S$. acidocaldarius and are consistent with the predominant mode of mutation observed in S. solfataricus (Jacobs and Grogan 1997, Martusewitsch et al. 2000). Analysis of the spontaneous mutational spectrum further shows that the S. acidocaldarius genome is the most stable of several microbial DNA genomes examined so far, and has an especially low rate of base-pair substitution (Grogan et al. 2001). Enteric bacteria in which MMR has been impaired by mutation or physiological stress (LeClerc et al. 1996, Harris et al. 1997) cannot approach the accuracy attained by wild-type $S$. acidocaldarius, even though this archaeon has a mildly acidic cytoplasm (about pH 6; Meyer and Schäfer 1992) and an optimal growth temperature of about $80^{\circ} \mathrm{C}$ (Grogan 1989).

The present study has demonstrated that accuracy-enforcing mechanisms of $S$. acidocaldarius can be inactivated by mutation, resulting in mutator strains that provide a basis for the experimental study of these mechanisms. Several mutators exhibited 50-fold or higher increases of forward mutation rates in the pyrE and pyrF genes. Control experiments showed that this result could not be attributed to increased EOP of these lineages on FOA medium or creation of a functional but genetically unstable $p y r E$ allele during isolation. Other mutant phenotypes unrelated to the FOA selection were also generated at elevated frequencies. These included ethionine-resistant mutants and phenotypic revertants of the pyrB4 allele. Reversion of pyrB 4 and other auxotrophic mutations has been shown to

Table 5. Sensitivity to DNA-damaging agents.

\begin{tabular}{|c|c|c|c|c|c|c|c|}
\hline \multirow[t]{2}{*}{ Candidate number } & \multirow[t]{2}{*}{ Mutagenized population } & \multirow{2}{*}{$\begin{array}{l}\text { cis-platin } \\
\left(\mathrm{MIC} ; \mu \mathrm{g} \mathrm{ml}{ }^{-1}\right)\end{array}$} & \multirow[t]{2}{*}{ Butadiene diepoxide } & \multirow[t]{2}{*}{ MNNG } & \multirow[t]{2}{*}{$\mathrm{MNU}$} & \multicolumn{2}{|c|}{ UV-C (percent survival) } \\
\hline & & & & & & $30 \mathrm{~J} \mathrm{~m}^{-2}$ & $60 \mathrm{~J} \mathrm{~m}^{-2}$ \\
\hline (DG64) & - & 60 & 150 & 150 & 5 & 21.6 & 0.16 \\
\hline (DG185) & - & 40 & 300 & 150 & 10 & 21.8 & 1.31 \\
\hline 2 & IV & 40 & 150 & 300 & 5 & 11.7 & 0.37 \\
\hline 7 & III & 40 & 150 & 300 & 5 & 16.5 & 1.55 \\
\hline 8 & I & 30 & 150 & 250 & 2.5 & 12.6 & 1.57 \\
\hline 10 & IV & 40 & 150 & 200 & 5 & 5.17 & 0.07 \\
\hline 11 & I & 55 & 150 & 150 & 5 & 18.0 & 0.43 \\
\hline 12 & III & 50 & 150 & 150 & 15 & -1 & -1 \\
\hline 13 & II & 20 & 150 & 50 & 10 & 17.4 & 1.22 \\
\hline 17 & III & 25 & 150 & 50 & 15 & 21.3 & 1.05 \\
\hline 19 & III & 40 & 150 & 100 & 15 & 29.6 & 0.43 \\
\hline 22 & III & 60 & 113 & 75 & 5 & 42.0 & 0.29 \\
\hline 23 & II & 20 & 150 & 100 & 5 & -1 & -1 \\
\hline 27 & IV & 60 & 150 & 100 & 15 & 16.0 & 0.17 \\
\hline
\end{tabular}

${ }^{1}$ Not determined. 
provide a sensitive assay of mutagenesis by UV-light (Wood et al. 1997) and other DNA-damaging agents in S. acidocaldarius (M. Reilly, University of Cincinnati and D. Grogan, unpublished observations). In several candidates, spontaneous formation of auxotrophic and Ts mutations, which were not selectable under our conditions, were also elevated.

We observed certain phenotypic differences among the candidates. For example, although it is not apparent in Table 1, Candidates 2 and 10 (both of which came from Population I) usually yielded much higher frequencies of $\mathrm{Foa}^{\mathrm{R}}$ mutants than the other candidates. Isolated $\mathrm{Oro}^{+}$colonies of these two strains typically gave confluent growth on FOA plates when streaked directly or when grown for a few generations in liquid medium before being spread (G. Bell, unpublished data), unlike the other strains we examined. Candidates 8 and 11 (both of which came from Population IV) differed from the others in yielding large numbers of Ts and auxotrophic mutants. The reason for failure to observe this in other candidates is unclear, but in principle it could reflect enhancement of a specific mutagenic mechanism. Frameshift mutations, for example, are much more common in the pyrE gene of $S$. acidocaldarius than the $p y r F$ gene largely because of the greater abundance of AT homopolymeric runs in pyrE (D. Grogan, unpublished observation). Frameshift mutations are also mechanistically unlikely to yield a Ts phenotype when introduced into protein-encoding genes.

In characterizing the candidates, we observed no obvious growth defects such as poor viability, low EOP, slow growth, or severe sensitivity to several DNA damaging agents. This suggests that, in hyperthermophilic archaea, as in eukarya and bacteria, at least some of the mechanisms that enforce genetic fidelity are not acutely important for cellular viability. In particular, the lack of any obvious sensitivity to killing or growth inhibition by DNA damaging agents suggests that the defects in the mutator strains do not seriously impede the repair of exogenous damage. This is reminiscent of most bacterial mutator mutants, whose defects usually have little impact on generalized DNA repair pathways (Shanabruch et al. 1982, Miller 1998).

The evidence that $S$. acidocaldarius (and presumably other hyperthermophilic archaea) carries out some form of mismatch correction that does not require MutS or MutL homo$\log$ s raises the question of the biochemical basis of such correction. Although completely novel mechanisms are possible, it seems likely that known DNA repair strategies, such as base-excision repair (BER), assume some of the functions that the MutS-MutL system carries out in other organisms. In E. coli, BER enzymes are more specialized in function than the MutS-MutL system, but nevertheless assist it in suppressing spontaneous mutation. For example, the ung gene product (uracil-N-glycosylase) of $E$. coli corrects the mutagenic effect of cytosine deamination by excising the resulting uracil from DNA, whereas the mutM gene product similarly removes mutagenic oxidized guanine residues (Miller 1998). Putative BER genes have been identified in genomes of hyperthermophilic archaea (Grogan 2000), and some BER enzymes of thermophilic archaea have activities relevant to MMR (Horst and Fritz 1996, Yang et al. 2000). It will thus be of interest to combine genetic and biochemical analyses in investigating which enzymes of hyperthermophilic archaea assume much of the MMR function normally played by MutS and MutL proteins in bacteria and eukarya.

\section{Acknowledgments}

This work was supported by National Science Foundation Grant MCB 9733303.

\section{References}

Aravind, L., D.R. Walker and E. Koonin. 1999. Conserved domains in DNA repair proteins and evolution of repair systems. Nucleic Acids Res. 27:1223-1242.

Bernander, R., A. Poplawski and D.W. Grogan. 2000. Altered patterns of cellular growth, morphology, replication and division in conditional-lethal mutants of the thermophilic archaeon Sulfolobus acidocaldarius. Microbiology 146:749-757.

Deckert, G., P. Warren, T. Gaasterland, et al. 1998. The complete genome of the hyperthermophilic bacterium Aquifex aeolicus. Nature 392:353-358.

Drake, J.W., B. Charlesworth, D. Charlesworth and J.F. Crow. 1998. Rates of spontaneous mutation. Genetics 148:1667-1686.

Eisen, J.A. 1998. A phylogenomic study of the MutS family of proteins. Nucleic Acids Res. 36:4291-4300.

Grogan, D.W. 1989. Phenotypic characterization of the archaebacterial genus Sulfolobus: comparison of five wild-type strains. J. Bacteriol. 171:6710-6719.

Grogan, D.W. 1995. Isolation of Sulfolobus acidocaldarius mutants. In Archaea: A Laboratory Manual. Eds. F.T. Robb and A.R. Place. Cold Spring Harbor Laboratory Press, Cold Spring Harbor, New York, pp 125-132.

Grogan, D.W. 2000. The question of DNA repair in hyperthermophilic archaea. Trends Microbiol. 8:180-185.

Grogan, D.W. and R.P. Gunsalus. 1993. Sulfolobus acidocaldarius synthesizes UMP via a standard de novo pathway: results of a biochemical-genetic study. J. Bacteriol. 175:1500-1507.

Grogan, D.W., G.T. Carver and J.W. Drake. 2001. Genetic fidelity under harsh conditions: Analysis of spontaneous mutation in the thermoacidophilic archaeon Sulfolobus acidocaldarius. Proc. Natl. Acad. Sci. 98:7928-7933.

Harris, R.S., G. Feng, K. Ross, R. Sidhu, C. Thulin, S. Longerich, S. Szigety, M. Winkler and S. Rosenburg. 1997. Mismatch repair protein MutL becomes limiting during stationary-phase mutation. Genes Dev. 11:2426-2437.

Horst, J.P. and H.J. Fritz. 1996. Counteracting the mutagenic effect of hydrolytic deamination of DNA 5-methylcytosine residues at high temperature: DNA mismatch N-glycosylase Mig.Mth of the thermophilic archaeon Methanobacterium thermoautotrophicum THF. EMBO J. 15:5459-5469.

Jacobs, K.L, and D.W. Grogan. 1997. Rates of spontaneous mutation in an archaeon from geothermal environments. J. Bacteriol. 179: 3298-3303.

LeClerc, J.E., B. Li, W. Payne and T.A. Cebula. 1996. High mutation frequencies among Escherichia coli and Salmonella pathogens. Science 274:1208-1211.

Lindahl, T. 1993. Instability and decay of the primary structure of DNA. Nature 362:709-715.

Mao, E.F., L. Lane, J. Lee and J.H. Miller. 1997. Proliferation of mutators in a cell population. J. Bacteriol. 179:417-722. 
Martusewitsch, E., C.W. Sensen and C. Schleper. 2000. High spontaneous mutation rate in the hyperthermophilic archaeon Sulfolobus solfataricus is mediated by transposable elements. J. Bacteriol. 182:2574-2581.

Meyer, W. and G. Schäfer. 1992. Characterization and purification of a membrane-bound archaebacterial pyrophophatase from Sulfolobus acidocaldarius. Eur. J. Biochem. 207:741-746.

Miller, J.H. 1998. Mutators in Escherichia coli. Mutat. Res. 409: 99-106.

Nakagawa, T., A. Datta and R. Kolodner. 1999. Multiple functions of MutS- and MutL-related heterocomplexes. Proc. Nat. Acad. Sci. 96:14,186-14,188.

Ng, W.V., S.P. Kennedy, G.G. Mahairas, et al. 2000. Genome sequence of Halobacterium species NRC-1. Proc. Natl. Acad. Sci. 97:12,176-12,181.

Nelson K.E., R.A. Clayton, S. Gill, et al. 1999. Evidence for lateral gene transfer between archaea and bacteria from genome sequence of Thermotoga maritima. Nature 399:323-329.

Nghiem, Y., M. Cabrera, C. Cupples and J.H. Miller. 1988. The mutY gene: a mutator locus in Escherichia coli that generates GC-to-TA transversions. Proc. Natl. Acad. Sci. 85:2709-2713.
Shanabruch, W.G., R.P. Rein, I. Behlau and G.C. Walker. 1982. Mutagenesis, by methylating agents, in mutH, mutL mutS, and $u v r D$ mutants of Salmonella typhimurium LT2. J. Bacteriol. 153:33-44.

Smith, D.R., L.A. Doucette-Stamm, C. Deloughery, et al. 1997. Complete genome sequence of Methanobacterium thermoautotrophicum $\Delta \mathrm{H}$ : Functional analysis and comparative genomics. J. Bacteriol. 179:7135-7155.

Sniegowski, P.D., P.J. Gerrish and R.E. Lenski. 1997. Evolution of high mutation rates in experimental populations of Escherichia coli. Nature 387:703-705.

Yang, H., S. Fitz-Gibbon, E.M. Marcotte, J.H. Tai, E.C. Hyman and J.H. Miller. 2000. Characterization of a thermostable DNA glycosylase specific for $\mathrm{U} / \mathrm{G}$ and $\mathrm{T} / \mathrm{G}$ mismatches from the hyperthermophilic archaeon Pyrobaculum aerophilum. J. Bacteriol. 182: 1272-1279.

Wood, E.R., F. Ghané and D.W. Grogan. 1997. Genetic responses of the thermophilic archaeon Sulfolobus acidocaldarius to shortwavelength UV light. J. Bacteriol. 179:5693-5698. 

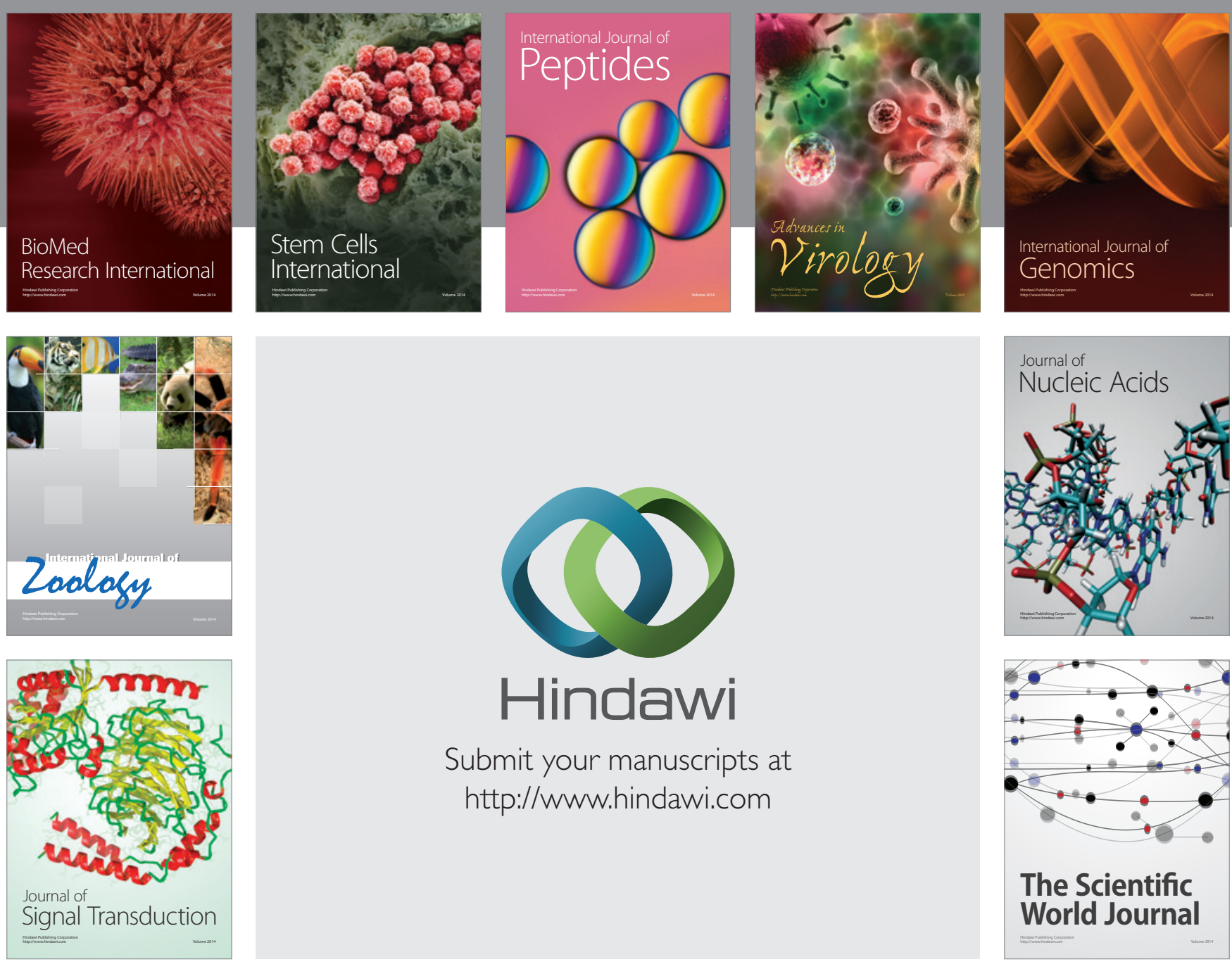

Submit your manuscripts at

http://www.hindawi.com
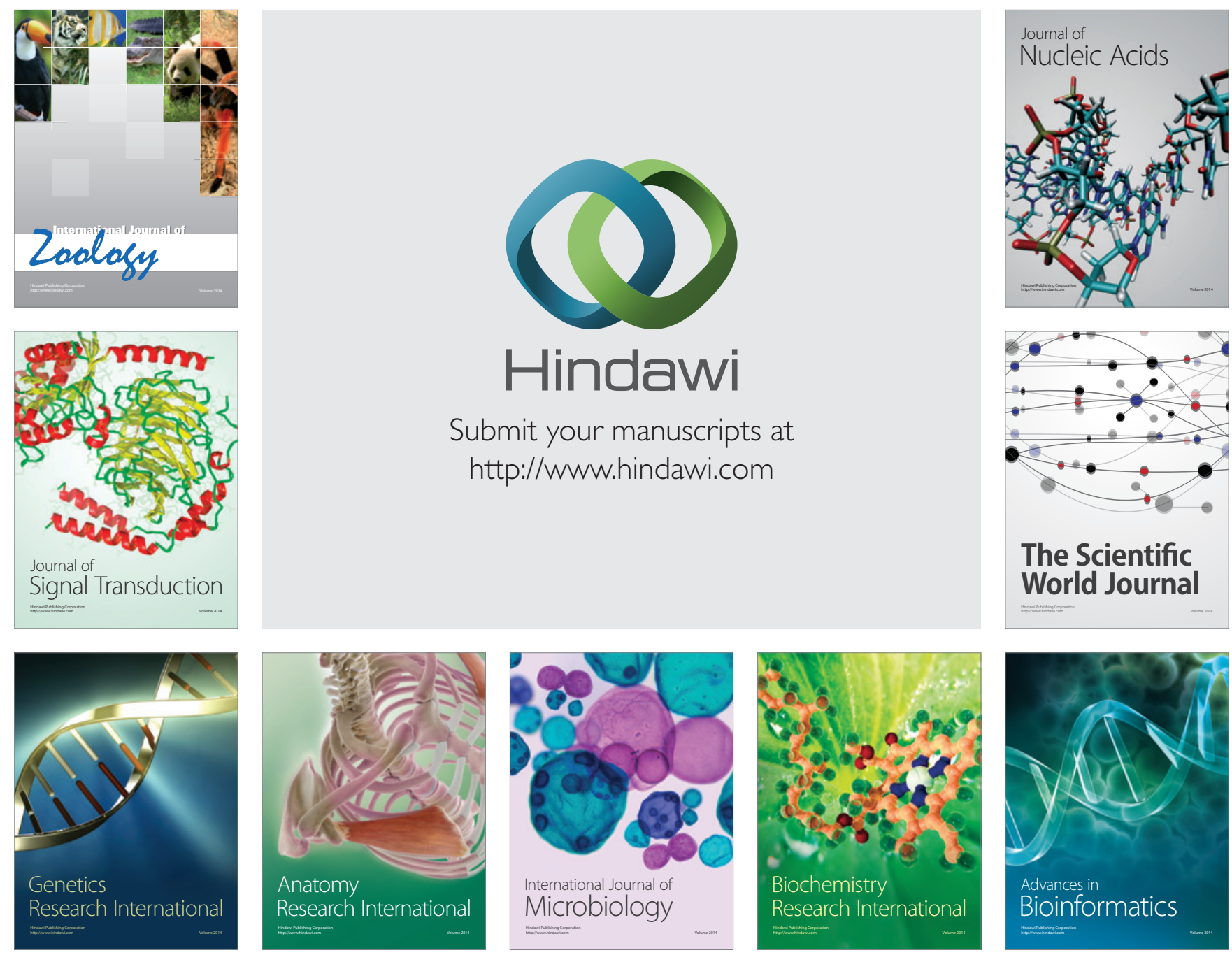

The Scientific World Journal
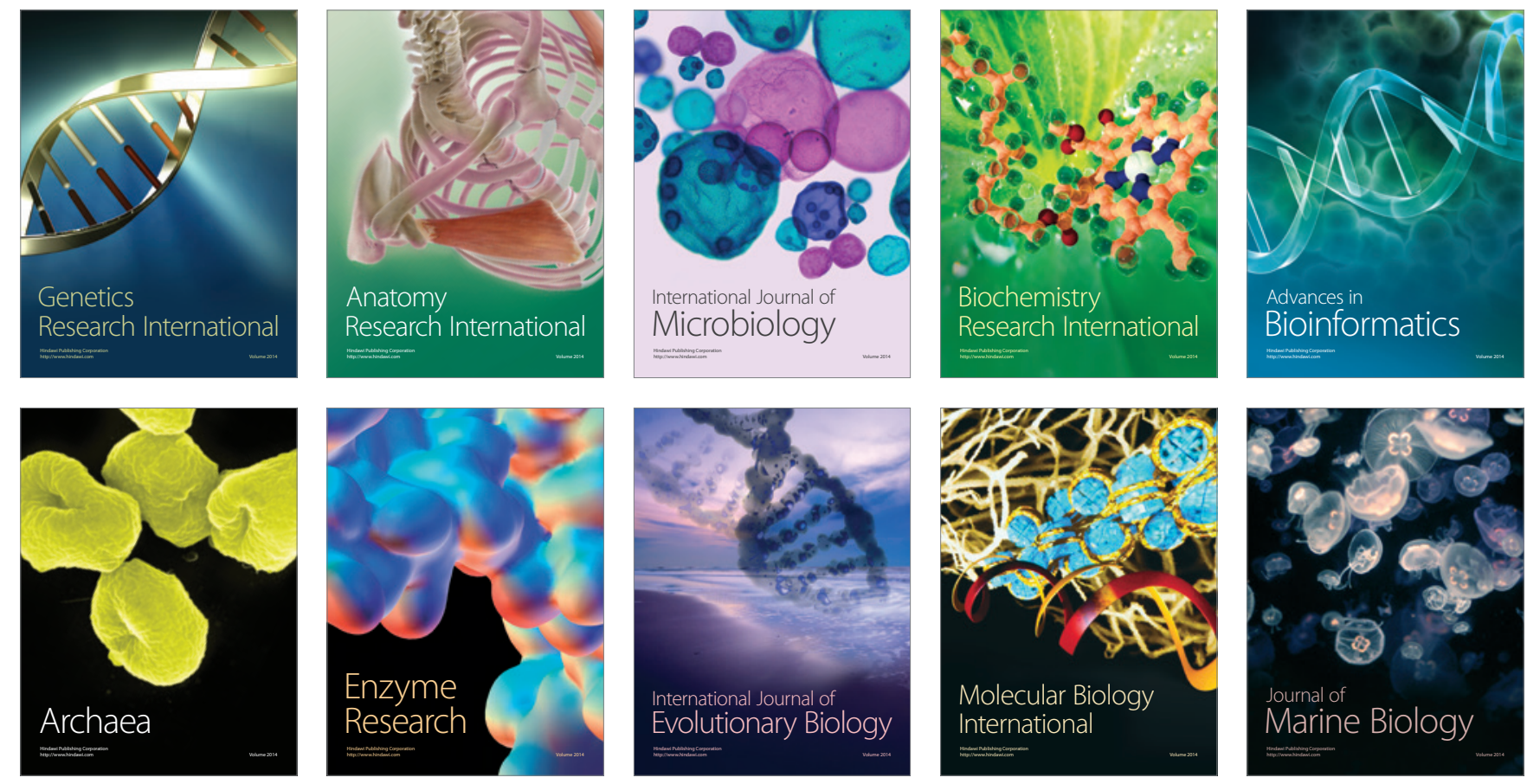